\title{
Update on optimal use of lisdexamfetamine in the treatment of ADHD
}

\author{
This article was published in the following Dove Press journal: \\ Neuropsychiatric Disease and Treatment \\ 19 July 2013 \\ Number of times this article has been viewed
}

\section{Vishal Madaan \\ Venkata Kolli \\ Durga P Bestha \\ Manan J Shah}

Department of Psychiatry and Neurobehavioral Sciences, Division of Child and Family Psychiatry, University of Virginia Health System, Charlottesville, VA, USA
Correspondence: Vishal Madaan Department of Psychiatry and Neurobehavioral Sciences, Division of Child and Family Psychiatry, University of Virginia Health System, PO Box 800623, Charlottesville,

VA 22908, USA

Tel +I 4342436950

Fax +I 4342436970

Emailvm8d@virginia.edu
Abstract: Lisdexamfetamine (LDX) has been a recent addition to the treatment armamentarium for Attention Deficit Hyperactivity Disorder (ADHD). It is unique among stimulants as it is a prodrug, and has been found to be safe and well-tolerated medication in children older than 6 years, adolescents and adults. It has a smooth onset of action, exerts its action up to 13 hours and may have less rebound symptoms. LDX has proven to be effective in the treatment of ADHD in placebo controlled trials, and improved performance in simulated academic and work environments have been noticed. Both stimulant-naïve and stimulant-exposed patients with ADHD appear to benefit from LDX. It has also shown some promise in improving emotional expression and executive function of patients with ADHD. Adverse effects such as decrease in sleep, loss of appetite and others have been reported with LDX use, just as with other stimulant formulations. Since most such studies exclude subjects with preexisting cardiac morbidity, prescribing precautions should be taken with LDX in such subjects, as with any other stimulant. Study subjects on LDX have been reported to have low scores on drug likability scales, even with intravenous use; as a result, LDX may have somewhat less potential for abuse and diversion. There is a need for future studies comparing other long acting stimulants with LDX in ADHD; in fact clinical trials comparing LDX with OROS (osmotic controlled-release oral delivery system) methylphenidate are currently underway. Furthermore, the utility of this medication in other psychiatric disorders and beyond ADHD is being investigated.

Keywords: lisdexamfetamine, ADHD, functional impairment, pharmacotherapy

\section{Introduction}

Attention deficit hyperactivity disorder (ADHD) is a neuropsychiatric condition characterized by problems with inattention, hyperactivity, and impulsivity, that result in functional impairment both at home and at school or work settings. Symptoms of ADHD often begin in early childhood, and persist well into adolescence and adulthood in about $50 \%-60 \%$ of individuals. ${ }^{1}$ ADHD is often associated with comorbid psychiatric disorders, such as oppositional defiant disorder, conduct disorder, and childhood mood and anxiety disorders. The worldwide prevalence of ADHD has been estimated to be $3 \%-7 \%$. $^{2}$ While some estimates suggest prevalence rates of ADHD to be around $7 \%-10 \%$, a recent systematic review using metaregression analysis indicated that the worldwide pooled prevalence of ADHD could be estimated at $5.29 \%$. $^{3}$

While pharmacotherapy appears to be the mainstay of treatment for ADHD, psychotherapy or a combination of both pharmacotherapy and psychotherapy are often used, especially in patients with comorbid psychiatric disorders. Pharmacologic treatments frequently used for ADHD include stimulants, nonstimulants such as atomoxetine, and 
the alpha- 2 agonists, clonidine and guanfacine. These can be used as either monotherapy, as an adjunct to another medication, or more recently, as combinations. ${ }^{3,4}$ Bupropion and modafinil are two other nonstimulant medications that have also been studied in ADHD, but have had limited success. ${ }^{5}$

The use of stimulants for inattention and hyperactivity symptoms was first described in the 1930s and was revisited in the 1960s with the frequent use of short-acting stimulant formulations. Multimodal treatment approaches, including pharmacotherapy, behavioral interventions, patient and parent education, and accommodation and utilization of school resources, constitute the core elements of treatment in ADHD. At present, both short-acting and long-acting formulations of stimulant medications, ie, methylphenidate and dextroamphetamine (d-amphetamine), are accepted as the first-line option for pharmacotherapy in ADHD. However, use of short-acting formulations is limited by the need for multiple dosing, the stigma of the school dose, and the higher risk of drug diversion. ${ }^{6}$

Based on concerns regarding abuse of stimulants, and utilizing a newer prodrug mechanism, lisdexamfetamine has been marketed as another longer-acting formulation of the dextroamphetamine group. As mentioned, it differs from other available extended-release stimulant formulations because of its mechanism of action as a prodrug which requires enzymatic breakdown before becoming the active, dextroamphetamine. ${ }^{7}$ Over the years, further studies have more accurately identified the site of the enzymatic breakdown. In this paper, we provide an overview of the recent literature on lisdexamfetamine, focusing on its pharmacology, safety, tolerability, and efficacy profiles.

\section{Pharmacologic aspects}

Lisdexamfetamine was first approved by the United States Food and Drug Administration for treatment of ADHD in children aged 6-12 years in 2007, and was subsequently approved for ADHD in adults in 2008 and then for adolescents in 2010. In February 2012, it was also approved as "maintenance for adults with ADHD". From a typical initial dose of $30 \mathrm{mg} /$ day, it can be uptitrated based on clinical response and tolerability to a maximum of $70 \mathrm{mg} /$ day in weekly increments of $10-20 \mathrm{mg} .{ }^{8}$ Lisdexamfetamine is currently the only stimulant prodrug available, with a unique formulation that may help to lower the potential for abuse, toxicity in cases of overdose, and the likelihood of drug tampering. ${ }^{9}$ Lisdexamfetamine contains the active ingredient dextroamphetamine and the amino acid lysine. Because it is a single enantiomer, it may have lesser propensity to cause adverse reactions compared with mixed-amphetamine extended-release salts (MAS XR, a 3:1 dextro:levo mixture). Lisdexamfetamine enters the portal circulation unchanged via the peptide protein transporter system, and the covalent linkage between lysine and dextroamphetamine is broken down by hydrolysis in the red blood cell. ${ }^{10}$ Krishnan and Moncrief demonstrated that lisdexamfetamine is not metabolized by the hepatic cytochrome P450 system, so lisdexamfetamine has a low potential for drug-drug interactions. ${ }^{11}$

The pharmacodynamics of dextroamphetamine include reuptake inhibition of monoamines and also direct stimulation of release of dopamine and norepinephrine. In healthy adult volunteers, a steady-state concentration of dextroamphetamine following administration of lisdexamfetamine $70 \mathrm{mg}$ was achieved by day $5 .{ }^{12}$ Consistent levels of mean peak plasma concentration $\left(\mathrm{C}_{\max }\right)$ and area under the curve for dextroamphetamine were recorded when healthy adult volunteers were administered lisdexamfetamine $50 \mathrm{mg}$ using oral capsules and InteliSite ${ }^{\mathrm{TM}}$ delivery capsules (Casper Associates, Raleigh, NC, USA) designed to release the drug selectively in selected small intestinal regions. ${ }^{13}$ Time to reach maximal concentration was delayed by one hour when $70 \mathrm{mg}$ of lisdexamfetamine was administered with food compared with the fasting state in healthy adult volunteers, but there was no change in $\mathrm{C}_{\max }$ levels. ${ }^{14}$ Absorption was not significantly affected whether lisdexamfetamine was administered with or without food or as a solution. ${ }^{9}$ A dose-proportional increase in the area under the curve and $\mathrm{C}_{\max }$ of dextroamphetamine with low interpatient variability was demonstrated in children with ADHD using doses of $30 \mathrm{mg}, 50 \mathrm{mg}$, and $70 \mathrm{mg} .{ }^{15}$ Oral and intranasal administration of lisdexamfetamine in healthy adult volunteers showed a similar $\mathrm{C}_{\max }$, area under the curve, and time to reach maximal concentration, with low intersubject variability and similar tolerability rates for both routes of administration. ${ }^{16}$

\section{Safety and tolerability}

As with the literature available on any relatively new pharmacologic agent, the available studies on lisdexamfetamine are limited by several factors, including predominantly pharmaceutical industry trials, shorter duration of randomized controlled trials, open-label designs for longer duration studies, and the unavailability of head-to-head trials with comparable agents, such as lisdexamfetamine versus extended-release methylphenidate or MAS XR studies. In terms of safety and tolerability, the common theme from the available studies is the presence of transient treatment emergent adverse events, including decreased appetite, decreased weight, irritability, insomnia, 
headache, upper abdominal pain, and initial insomnia, which improve over time. No statistically significant changes in vital signs, laboratory work, and electrocardiographic readings have been noted. Overall, lisdexamfetamine has been demonstrated to be well tolerated in child-adolescent and adult populations. However, it should be noted that most of the available studies exclude subjects with psychiatric and general medical comorbidities. Because of this limitation, one should be careful when prescribing any stimulants, including lisdexamfetamine, because there may be a potential for increased risk with already diagnosed or masked comorbid illnesses, such as tic disorder or hypertension, or if the patient has a significant family history of cardiovascular disease. Further, it should be noted that the safety and efficacy of lisdexamfetamine in patients aged 3-5 years has not been established.

Biederman et $\mathrm{al}^{17}$ performed a randomized, controlled Phase II trial including 52 children aged 6-12 years with ADHD in an analog classroom setting. Following an initial 3-day washout period, all subjects were treated with MAS $\mathrm{XR}$, which was titrated starting at $10 \mathrm{mg}$ and increased up to $30 \mathrm{mg}$ based on response, over a period of 3 weeks. After the dose-titration phase, the subjects entered the randomized, three-arm, crossover phase during which the subjects were treated with MAS XR, lisdexamfetamine, and placebo for one week each. The effective MAS XR dose determined during the initial titration period was used in the MAS XR arm and the equivalent dose of lisdexamfetamine (30-70 $\mathrm{mg}$ ) was used in the lisdexamfetamine cohort. There was a statistically significant improvement $(P<0.001)$ in both the MAS XR and lisdexamfetamine groups. This was measured using the Swanson, Kotkin, Agler, M-Flynn, and Pelham (SKAMP), Permanent Product Measure of Performance (PERMP), and the Clinical Global Impression (CGI) rating scales. Insomnia, decreased appetite, and anorexia were the most common adverse effects noticed with lisdexamfetamine. Small changes in diastolic blood pressure and pulse rate, along with changes in the QTc interval, were noted but were not clinically or statistically significant. Limitations of this study included its small sample size, the possibility of improved tolerability due to the initial dose-titration phase with MAS XR, exclusion of children with psychiatric comorbidities, and not comparing lisdexamfetamine head-to-head with MAS XR.

A forced-dose, randomized, controlled Phase III trial conducted by Biederman et $\mathrm{al}^{18}$ included 290 children (6-12 years) with ADHD who were randomized into four cohorts. The first cohort received placebo, the second cohort received $30 \mathrm{mg}$ of lisdexamfetamine for 4 weeks, the third cohort received $30 \mathrm{mg}$ of lisdexamfetamine for the first week followed by $50 \mathrm{mg}$ for the next 3 weeks, and the fourth cohort was treated with $30 \mathrm{mg}$ of lisdexamfetamine for the first week, $50 \mathrm{mg}$ for the next week, then $70 \mathrm{mg}$ for the next 2 weeks. Compared with the placebo group, ADHD Rating Scale Version IV (ADHD-RS-IV) score reductions were $4-5$-fold $(P<0.001)$ greater in the lisdexamfetamine groups, with the largest reductions recorded in the $70 \mathrm{mg}$ lisdexamfetamine group. Consistent improvements were also noted on the Conner's Parent Rating Scale-Revised Short Form at 10 am, 2 pm, and 6 pm. Adverse effects were reported mainly during the first week of treatment, and included decreased appetite, weight loss, insomnia, upper abdominal pain, headache, irritability, nausea, and vomiting. These resolved over the course of the study for the majority of subjects. However, $9 \%$ of the subjects withdrew due to adverse effects. There were no significant changes in the subjects' electrocardiograms, but there was a mild increase in heart rate of 4-5 beats per minute.

Findling et $\mathrm{l}^{19}$ then followed 272 children from the above two studies. After an initial 4-week treatment responsebased dose-titration period starting with lisdexamfetamine doses of $30 \mathrm{mg}$ and going up to $70 \mathrm{mg}$, the subjects entered an 11-month dose-maintenance phase. Improvements in ADHD-RS-IV scores started at one week and were sustained throughout the study. Lisdexamfetamine was well tolerated, and the only adverse effects were insomnia and irritability, reported by more than $5 \%$ of the subjects. The risk of adverse cardiovascular effects remains a concern with stimulant medications. This was studied by Hammerness et $\mathrm{al}^{20}$ in their prospective open-label study of 15 patients with ADHD. Lisdexamfetamine was titrated to $70 \mathrm{mg}$ over 6 weeks, and these individuals were then followed for 6 months on the $70 \mathrm{mg}$ dose. Transthoracic echocardiography and cardiopulmonary exercise testing showed changes in resting left ventricular systolic function and diastolic indices on treatment with lisdexamfetamine. However, these changes were not in the direction of cardiomyopathy and there were no major changes in cardiac function at maximal exertion. Interestingly, heart rate recovery was elevated by 4 beats per minute in this study. ${ }^{20}$ Mora et al suggest that impaired heart rate recovery may be an indicator of cardiovascular risk. ${ }^{21}$

In another study by Findling et al, 318 children (6-12 years) with ADHD entered an open-label, 7-week, dose-optimization study with a starting lisdexamfetamine dose of $20 \mathrm{mg}$, with $10 \mathrm{mg}$ dose increments at weekly intervals up to a maximum dose of $70 \mathrm{mg}$ based on response following a 2-week washout period. ${ }^{22}$ Lisdexamfetamine was well tolerated throughout this study. Statistically significant 
$(P<0.001)$ improvements on the ADHD-RS-IV scale were found, as well as improvements on the CGI, Parent Global Assessment, Behavior Rating Inventory of Executive Function (BRIEF), and Expression and Emotion Scale for Children (EESC) scales. Two patients experienced serious adverse events during this trial. The first occurred in a 12-year-old girl with a prior history of intermittent syncope who experienced a syncopal episode on lisdexamfetamine $60 \mathrm{mg}$ that was considered a serious adverse event, but she went on to complete the study successfully. The second serious adverse event occurred in a 7-year-old boy with an implanted loop recorder after a prior near-drowning incident, who was noted to have a sinus arrest of 6 seconds while on lisdexamfetamine $30 \mathrm{mg}$. Although this was determined to be unrelated to lisdexamfetamine, the subject was discontinued from the study. Despite these two serious adverse events, high levels of satisfaction with medication were reported by parents on the Medication Satisfaction Questionnaire during the trial.

Wigal et $\mathrm{al}^{23}$ conducted a small 4-week, open-label trial of lisdexamfetamine in 13 stimulant-naïve and 14 stimulant-exposed ADHD children. While the duration of the treatment emergent adverse events experienced in both groups was similar, the stimulant-naïve group reported more adverse events, including difficulty sleeping, abdominal pain, and "hyperfocus". Concern about disruption of sleep exists with any stimulant option. Giblin et al conducted a study involving 24 ADHD subjects aged 6-12 years to investigate the effect of lisdexamfetamine using latency to persistent sleep as the primary outcome, and the readings from polysomnography and an actigraph as secondary outcome measures. After an initial washout period followed by open-label dose optimization with a lisdexamfetamine dose range of $30-70 \mathrm{mg}$, the subjects were randomized to optimal dose and placebo groups. Lisdexamfetamine was not associated with sleep disturbances on either subjective or objective measures, and sleep efficiency was reported as being slightly improved compared with the placebo group. ${ }^{24}$

Another common concern with stimulant medications in children is growth retardation. In a 15-month follow-up of 282 children treated with lisdexamfetamine, Faraone et $\mathrm{al}^{25}$ used weight, height, and body mass index to show that there was slowing of growth on all the above parameters when compared with Centers for Disease Control norms. This delay was associated with the children being stimulant-naïve, heavier and taller on entry into the study, and having "greater cumulative exposure" to medication. Results from this study and similar research findings highlight the importance of using growth charts and enlisting parents/caregivers to monitor nutrition intake and growth charts closely.

\section{Efficacy}

The efficacy of lisdexamfetamine has been examined in comparison with placebo in several trials. In a 4-week, randomized, controlled trial ${ }^{26}$ involving 314 adolescents with ADHD who were randomized to receive placebo or to be part of a forced titration arm of lisdexamfetamine (30 mg, $50 \mathrm{mg}$, and $70 \mathrm{mg}$ ), lisdexamfetamine did well across all of the doses in comparison with placebo, as evidenced by improvement in scores on the ADHD-RS-IV, CGI, and Youth Quality of Life-Research Version. In a randomized, placebo-controlled trial of 420 adult subjects with ADHD, Adler et $\mathrm{al}^{27}$ found statistically significant improvements in ADHD-RS-IV and CGI scores for all doses of lisdexamfetamine (30-50 mg) compared with placebo. These improvements were evident from the first week. Six percent of subjects in the lisdexamfetamine group discontinued the drug owing to side effects, the most common being insomnia. Palpitations, hypertension, and dyspnea were also more common in the treatment group than in the placebo group. Weisler et $\mathrm{al}^{28}$ recruited 349 patients from the study by Adler et $\mathrm{al}^{27}$ for a 12-month, follow-up study, which was completed by 191 patients. The statistically significant improvement in ADHD-RS-IV score found in the previous study was maintained throughout the follow-up study. A post hoc analysis of this study by Mattingly et $\mathrm{al}^{29}$ showed that lisdexamfetamine was effective for all the subtypes of ADHD. Brams et $\mathrm{l}^{30}$ used a novel design of randomized withdrawal, in which 116 adult patients with ADHD and stable on lisdexamfetamine were randomized to placebo or lisdexamfetamine. Their results showed that most of the patients switched to the placebo group relapsed within 2 weeks. In an open-label study of 40 adults treated with lisdexamfetamine for 12 weeks, Adler et $\mathrm{al}^{31}$ found a significant reduction in ADHD-RS-IV scores. Based on assessments using the Time-Sensitive ADHD Symptom Scale, they asked patients to rate their ADHD symptoms a few hours earlier, and a significant improvement was reported. They also found that lisdexamfetamine had a smooth medication effect throughout the day, with fewer rebound symptoms. However, insomnia was a common adverse effect and one patient could not tolerate a dose increase for this reason. It was also reported that six patients withdrew from the study due to side effects. Jain et $\mathrm{al}^{32}$ performed a post hoc analysis of two large studies in children aged 6-12 years who were switched from methylphenidate to lisdexamfetamine. Sixty seven out of 129 subjects in the study by Wigal et $\mathrm{al}^{33}$ and 83 subjects out of 318 participants in the study by Findling et $\mathrm{al}^{22}$ received treatment with methylphenidate in the 6 months preceding the lisdexamfetamine 
trail, and these subjects were included in the subgroup analysis. The subgroup with prior methylphenidate treatment showed significant improvement in ADHD-RS-IV scores, which were comparable with overall results from the two open-label trials.

\section{Effect on performance in academic and work environments}

One of the important benefits of treating children with ADHD is an improvement in their academic performance. This improvement has been studied in several clinical trials. In a "13-hour laboratory school" study following a 4-week, dose-optimization phase of lisdexamfetamine (30-70 mg) involving 117 children (6-12 years) with ADHD, Wigal et al ${ }^{33}$ conducted a randomized, two-way crossover study of lisdexamfetamine and placebo with each arm lasting one week. Statistically significant improvements were noted on the SKAMP deportment rating, SKAMP attention, and Permanent Product Measure of Performance scales $(P<0.001)$. In another open-label, dose-optimization study of lisdexamfetamine $30-70 \mathrm{mg}$ in 26 children with ADHD, Wigal et al ${ }^{34}$ demonstrated improvements in rate, accuracy, and comprehension measures on the Gray Oral Reading Test-4 performed at baseline and after 4-5 weeks of treatment in a "modified laboratory school" setting. In a 7-week, open-label study of 319 children (6-12 years) with ADHD, Turgay et a ${ }^{35}$ found significant improvements in ADHD-RS-IV, BRIEF, and EESC scores. The first 5 weeks of the study were a dose-optimization phase (lisdexamfetamine $20-70 \mathrm{mg}$ ) and the final 2 weeks were a dose-maintenance phase. In a post hoc analysis of this 7-week, open-label lisdexamfetamine study, Katic et a ${ }^{36}$ used the clinically meaningful change and clinically significant/ reliable change categories derived from the EESC to show that there was no deterioration in emotional expression in a majority of subjects when taking lisdexamfetamine. However, it should be noted that the original study was limited by exclusion of patients with psychiatric comorbidity.

Other research that examined the efficacy of lisdexamfetamine in an academic or work environment includes a small open-label study Brown et $\mathrm{a}^{37}$ that showed a statistically significant improvement in executive functioning in ADHD subjects treated with lisdexamfetamine using the Brown Attention-Deficit Disorder Scale. In a simulated workplace environment, Wigal et a ${ }^{138}$ noted a consistent improvement in Permanent Product Measure of Performance scores in ADHD subjects treated with lisdexamfetamine in comparison with placebo. Use of lisdexamfetamine in patients with ADHD may improve functioning in academic and work environments, but the level of functioning may still not be equal to that of their non-ADHD peers. In an open-label study, Dupaul et a ${ }^{39}$ examined 24 college students with ADHD over a 5-week period using placebo and lisdexamfetamine $30 \mathrm{mg}, 50 \mathrm{mg}$, and $70 \mathrm{mg}$. Twenty-six healthy control subjects also participated in the study. Weekly measures, including the Conners Adult ADHD Rating Scales-Short Form, BRIEF-Adult Version, EESC, and Conners Continuous Performance Test California Verbal Learning Test-Second Edition, were administered. Even though an $86 \%$ improvement in ADHD symptoms was reported, along with improvement in executive functioning and small changes in psychosocial functioning, there remained a gap in comparison with control subjects.

\section{Effect on driving performance}

Appropriate treatment of ADHD may improve not only academic performance but also driving performance in licensed drivers. In a randomized, controlled trial involving 61 ADHD subjects aged $18-26$ years, Biederman et al ${ }^{40}$ showed that lisdexamfetamine-treated patients had a lower rate of collisions and faster reaction time in comparison with placebo in a validated driving simulation paradigm.

\section{Potential for misuse and abuse}

Patients with ADHD have a high risk for comorbidity involving substance misuse disorders, so it is imperative to evaluate the potential for an ADHD medication to lead to abuse or dependence. In an open-label lisdexamfetamine study with an innovative design, Donovan and Levin ${ }^{41}$ treated eight adolescents with ADHD whose older siblings had substance misuse problems. In addition to improvement in ADHD symptoms, the subjects also showed an improvement on the modified Pretcher and Nash scale which measures perceived benefit of substance abuse. Drug accountability records showed that

Table I Registered trials with lisdexamfetamine

Effects on the cardiovascular system: NCT007530I2

Treatment of cocaine dependence: NCT0I4902I6, NCTOI4868I0, NCT00958282

Attention deficit hyperactivity disorder (ADHD) smoking cessation study: NCT00736255

Major depressive disorder: NCTOI I48979, NCT0I436I49,

NCT0I436I62, NCT00905424, NCT00985725, NCT0I436I75

Bipolar depression: NCTOI I 31559, NCTOI093963

Cognitive dysfunction in multiple sclerosis: NCTO1615887

Attention and memory impairments in menopausal women: NCTOI 324024

Traumatic brain injury (TBI)-related attention deficits: NCTOI000064 Lisdexamfetamine versus other stimulants: NCTOI 106430 ,

NCT0076397, NCT01552915, NCT00889915

Binge eating disorder: NCT0I0907I3, NCTOI29I I73, NCT0I6570I9

Schizophrenia: NCT0I457339, NCT00922272, NCT0I738698 
$90 \%$ of the unreturned medication was "presumably having taking the pills as prescribed".

Lisdexamfetamine is hypothesized to have less abuse potential because of its formulation as a prodrug; however, it is still a controlled substance and it is very important to monitor the risk of possible diversion and abuse. Because of this risk, research has been done to examine the potential for lisdexamfetamine to be misused or abused. A randomized, three-arm crossover study ${ }^{42}$ of intravenous administration of lisdexamfetamine $25 \mathrm{mg}$ or $50 \mathrm{mg}$, immediate-release dextroamphetamine $10 \mathrm{mg}$ or $20 \mathrm{mg}$, or placebo in 12 healthy subjects with a history of stimulant abuse showed that lisdexamfetamine was not different from placebo in "liking effects" whereas immediate-release dextroamphetamine was associated with euphoria within 15 minutes. A randomized, six-step, crossover Phase I study ${ }^{43}$ in individuals with a history of stimulant abuse using placebo, lisdexamfetamine $50 \mathrm{mg}, 100 \mathrm{mg}$, and $150 \mathrm{mg}$, immediate-release dextroamphetamine $40 \mathrm{mg}$, diethylpropion $200 \mathrm{mg}$, and placebo showed that, compared with placebo, dextroamphetamine and diethylpropion produced significant differences on the Drug Rating Questionnaire-Subject Liking Scale. The $50 \mathrm{mg}$ and $100 \mathrm{mg}$ doses of lisdexamfetamine were similar to placebo; however, the $150 \mathrm{mg}$ dose was equivalent to the $40 \mathrm{mg}$ dose of dextroamphetamine.

\section{Conclusion}

The economic impact of ADHD in the United States has been estimated to be around 31.6 billion dollars, of which only 1.2 billion can be explained by the treatment costs directly related to ADHD. The rest can be attributed to a lack of productivity resulting from ADHD and other health care expenses incurred by ADHD patients and their families. ${ }^{44}$ Pharmacotherapy for ADHD is an efficacious as well as cost-effective treatment measure for ADHD when compared with behavioral therapy alone and compared with combined use of behavioral and pharmacotherapy. ${ }^{45}$ More research is required to study the individual pharmacotherapeutic options, including nonstimulants. In particular, it will be interesting to see the results of head-to-head studies of longacting stimulant options. It will also be interesting to see the emergence of data from studies looking at real-life ADHD subjects where there is no absolute exclusion of comorbid conditions. Lisdexamfetamine has shown to be safe, well tolerated, and efficacious in the management of ADHD in the child-adolescent and adult populations. The utility of lisdexamfetamine beyond ADHD is also being investigated. In a recent double-blind, placebo-controlled study of 26 patients, Young ${ }^{46}$ showed an improvement in both fatigue and executive function in patients with chronic fatigue syndrome.
Other ongoing studies (Table 1) are looking at the potential role of lisdexamfetamine in treatment-refractory depression, narcolepsy, traumatic brain injury, and other disorders.

\section{Disclosure}

Dr Madaan has received research support from Pfizer, Lilly, Otsuka, Merck, Shire and Forest. He has also served as a consultant for the NOW coalition for bipolar disorder. None of the other authors have any conflicts of interest in this work.

\section{References}

1. Turgay A, Goodman DW, Asherson P, et al. Lifespan persistence of ADHD: the life transition model and its application. J Clin Psychiatry. 2012;73(2):192-201.

2. Pliszka SR. Attention-deficit/hyperactivity disorder. In: Dulcan's Textbook of Child and Adolescent Psychiatry, 1st ed. Arlington, VA: American Psychiatric Publishing Inc; 2010. Available from http://www. ap.psychiatryonline.org/content.aspx?bookid=24\&sectionid=1311465 . Accessed June 13, 2013.

3. Polanczyk G, de Lima MS, Horta BL, Biederman J, Rohde LA. The worldwide prevalence of ADHD: a systematic review and metaregression analysis. Am J Psychiatry. 2007;164(6):942-948.

4. Brown RT, Amler RW, Freeman WS, et al. Treatment of attentiondeficit/hyperactivity disorder: overview of the evidence. Pediatrics. 2005;115(6):e749-e757.

5. Daughton JM, Kratochvil CJ. Review of ADHD pharmacotherapies: advantages, disadvantages, and clinical pearls. J Am Acad Child Adolesc Psychiatry. 2009;48(3):240-248.

6. Cascade E, Kalali AH, Weisler RH. Short-acting versus long-acting medications for the treatment of ADHD. Psychiatry (Edgmont). 2008;5(8):24-27

7. Madaan V. Lisdexamfetamine dimesylate for childhood ADHD. Drugs Today (Barc). 2008;44(5):319-324.

8. Domnitei D, Madaan V. New and extended-action treatments in the management of ADHD: a critical appraisal of lisdexamfetamine in adults and children. Neuropsychiatr Dis Treat. 2010;6:273-279.

9. Goodman DW. Lisdexamfetamine dimesylate: the first prodrug stimulant. Psychiatry (Edgmont). 2007;4(8):39-45.

10. Pennick M. Absorption of lisdexamfetamine dimesylate and its enzymatic conversion to d-amphetamine. Neuropsychiatr Dis Treat. 2010;6: 317-327.

11. Krishnan S, Moncrief S. An evaluation of the cytochrome p450 inhibition potential of lisdexamfetamine in human liver microsomes. Drug Metab Dispos. 2007;35(1):180-184.

12. Krishnan SM, Stark JG. Multiple daily-dose pharmacokinetics of lisdexamfetamine dimesylate in healthy adult volunteers. Curr Med Res Opin. 2008;24(1):33-40.

13. Ermer JC, Haffey MB, Doll WJ, et al. Pharmacokinetics of lisdexamfetamine dimesylate after targeted gastrointestinal release or oral administration in healthy adults. Drug Metab Dispos. 2012;40(2):290-297.

14. Krishnan S, Zhang Y. Relative bioavailability of lisdexamfetamine 70-mg capsules in fasted and fed healthy adult volunteers and in solution: a single-dose, crossover pharmacokinetic study. J Clin Pharmacol. 2008;48(3):293-302.

15. Boellner SW, Stark JG, Krishnan S, Zhang Y. Pharmacokinetics of lisdexamfetamine dimesylate and its active metabolite, d-amphetamine, with increasing oral doses of lisdexamfetamine dimesylate in children with attention-deficit/hyperactivity disorder: a single-dose, randomized, open-label, crossover study. Clin Ther. 2010;32(2):252-264.

16. Ermer JC, Dennis K, Haffey MB, et al. Intranasal versus oral administration of lisdexamfetamine dimesylate: a randomized, open-label, two-period, crossover, single-dose, single-centre pharmacokinetic study in healthy adult men. Clin Drug Investig. 2011;31(6): 357-370. 
17. Biederman J, Boellner SW, Childress A, Lopez FA, Krishnan S, Zhang Y. Lisdexamfetamine dimesylate and mixed amphetamine salts extendedrelease in children with ADHD: a double-blind, placebo-controlled, crossover analog classroom study. Biol Psychiatry. 2007;62(9): 970-976.

18. Biederman J, Krishnan S, Zhang Y, McGough JJ, Findling RL. Efficacy and tolerability of lisdexamfetamine dimesylate (NRP-104) in children with attention-deficit/hyperactivity disorder: a phase III, multicenter, randomized, double-blind, forced-dose, parallel-group study. Clin Ther. 2007;29(3):450-463.

19. Findling RL, Childress AC, Krishnan S, McGough JJ. Long-term effectiveness and safety of lisdexamfetamine dimesylate in school-aged children with attention-deficit/hyperactivity disorder. CNS Spectr. 2008;13(7):614-620.

20. Hammerness P, Zusman R, Systrom D, et al. A cardiopulmonary study of lisdexamfetamine in adults with attention-deficit/hyperactivity disorder World J Biol Psychiatry. 2013;14(4):299-306.

21. Mora S, Redberg RF, Cui Y, et al. Ability of exercise testing to predict cardiovascular and all-cause death in asymptomatic women: a 20 -year follow-up of the lipid research clinics prevalence study. JAMA. 2003;24;290(12):1600-1607.

22. Findling RL, Ginsberg LD, Jain R, Gao J. Effectiveness, safety, and tolerability of lisdexamfetamine dimesylate in children with attention-deficit/ hyperactivity disorder: an open-label, dose-optimization study. J Am Acad Child Adolesc Psychiatry. 2009;19(6):649-662.

23. Wigal SB, Wong AA, Jun A, Stehli A, Steinberg-Epstein R, Lerner MA. Adverse events in medication treatment-naïve children with attentiondeficit/hyperactivity disorder: results from a small, controlled trial of lisdexamfetamine dimesylate. J Child Adolesc Psychopharmacol. 2012;22(2):149-156.

24. Giblin JM, Strobel AL. Effect of lisdexamfetamine dimesylate on sleep in children with ADHD. J Atten Disord. 2011;15(6):491-498.

25. Faraone SV, Spencer TJ, Kollins SH, Glatt SJ. Effects of lisdexamfetamine dimesylate treatment for ADHD on growth. J Am Acad Child Adolesc Psychiatry. 2010;49(1):24-32.

26. Findling RL, Childress AC, Cutler AJ, et al. Efficacy and safety of lisdexamfetamine dimesylate in adolescents with attentiondeficit/hyperactivity disorder. J Am Acad Child Adolesc Psychiatry. 2011;50(4):395-405.

27. Adler LA, Goodman DW, Kollins SH, et al. Double-blind, placebo-controlled study of the efficacy and safety of lisdexamfetamine dimesylate in adults with attention-deficit/hyperactivity disorder. J Clin Psychiatry. 2008;69(9):1364-1373.

28. Weisler R, Young J, Mattingly G, et al. Long-term safety and effectiveness of lisdexamfetamine dimesylate in adults with attention-deficit/ hyperactivity disorder. CNS Spectr. 2009;14(10):573-585.

29. Mattingly G, Weisler R, Dirks B, et al. Attention deficit hyperactivity disorder subtypes and symptom response in adults treated with lisdexamfetamine dimesylate. Innov Clin Neurosci. 2012;9(5-6):22-30.

30. Brams M, Weisler R, Findling RL, et al. Maintenance of efficacy of lisdexamfetamine dimesylate in adults with attention-deficit/hyperactivity disorder: randomized withdrawal design. J Clin Psychiatry. 2012;73(7):977-983

31. Adler LA, Lynch LR, Shaw DM, et al. Effectiveness and duration of effect of open-label lisdexamfetamine dimesylate in adults with ADHD. JAtten Disord. May 8, 2013. [Epub ahead of print.]
32. Jain R, Babcock T, Burtea T, et al. Efficacy and safety of lisdexamfetamine dimesylate in children with attention-deficit/hyperactivity disorder and recent methylphenidate use. Adv Ther. May 17, 2013. [Epub ahead of print.]

33. Wigal SB, Kollins SH, Childress AC, Squires L. A 13-hour laboratory school study of lisdexamfetamine dimesylate in school-aged children with attention-deficit/hyperactivity disorder. Child Adolesc Psychiatry Ment Health. 2009;3(1):17.

34. Wigal SB, Maltas S, Crinella F, et al. Reading performance as a function of treatment with lisdexamfetamine dimesylate in elementary school children diagnosed with ADHD. J Atten Disord. 2012;16(1):23-33.

35. Turgay A, Ginsberg L, Sarkis E, et al. Executive function deficits in children with attention-deficit/hyperactivity disorder and improvement with lisdexamfetamine dimesylate in an open-label study. J Child Adolesc Psychopharmacol. 2010;20(6):503-511.

36. Katic A, Ginsberg L, Jain R, et al. Clinically relevant changes in emotional expression in children with ADHD treated with lisdexamfetamine dimesylate. J Atten Disord. 2012;16(5):384-397.

37. Brown TE, Brams M, Gao J, Gasior M, Childress A. Open-label administration of lisdexamfetamine dimesylate improves executive function impairments and symptoms of attention-deficit/hyperactivity disorder in adults. Postgrad Med. 2012;122(5):7-17.

38. Wigal T, Brams M, Gasior M, et al. Randomized, double-blind, placebocontrolled, crossover study of the efficacy and safety of lisdexamfetamine dimesylate in adults with attention-deficit/hyperactivity disorder: novel findings using a simulated adult workplace environment design. Behav Brain Funct. 2012;6(1):34.

39. Dupaul GJ, Weyandt LL, Rossi JS, et al. Double-blind, placebocontrolled, crossover study of the efficacy and safety of lisdexamfetamine dimesylate in college students with ADHD. J Atten Disord. 2012;16(3):202-220.

40. Biederman J, Fried R, Hammerness P, et al. The effects of lisdexamfetamine dimesylate on the driving performance of young adults with ADHD: a randomized, double-blind, placebo-controlled study using a validated driving simulator paradigm. J Psychiatr Res. 2012;46(4):484-491.

41. Donovan SJ, Levin FR. The "younger-sibling-at-risk design": a pilot study of adolescents with ADHD and an older sibling with substance use disorder. Am J Drug Alcohol Abuse. 2011;37(40):235-239.

42. Jasinski DR, Krishnan S. Human pharmacology of intravenous lisdexamfetamine dimesylate: abuse liability in adult stimulant abusers. $J$ Psychopharmacol. 2009;23(4):410-418.

43. Weber J, Siddiqui MA. Lisdexamfetamine dimesylate: in attention-deficit hyperactivity disorder in adults. CNS Drugs. 2009;23(5):419-425.

44. Birnbaum HG, Kessler RC, Lowe SW. Costs of attention deficithyperactivity disorder (ADHD) in the US: excess costs of persons with ADHD and their family members in 2000. Curr Med Res Opin. 2005;21(2):195-206.

45. Wu EQ, Hodgkins P, Ben-Hamadi R, et al. Cost effectiveness of pharmacotherapies for attention-deficit hyperactivity disorder: a systematic literature review. CNS Drugs. 2012;26(7):581-600.

46. Young JL. Use of lisdexamfetamine dimesylate in treatment of executive functioning deficits and chronic fatigue syndrome: a double blind, placebo-controlled study. Psychiatry Res. 2013;207(1-2):127-133.
Neuropsychiatric Disease and Treatment

\section{Publish your work in this journal}

Neuropsychiatric Disease and Treatment is an international, peerreviewed journal of clinical therapeutics and pharmacology focusing on concise rapid reporting of clinical or pre-clinical studies on a range of neuropsychiatric and neurological disorders. This journa is indexed on PubMed Central, the 'PsycINFO' database and CAS

\section{Dovepress}

The manuscript management system is completely online and includes a very quick and fair peer-review system, which is all easy to use. Visit http://www.dovepress.com/testimonials.php to read real quotes from published authors. 